\title{
A curva de distribuição granulométrica e sua influência na reologia de concretos refratários
}

\section{(The particle size distribution effect on the rheology of refractory castables)}

\author{
F. T. Ramal Jr ${ }^{1}$, R. G. Pileggi ${ }^{1}$, J. B. Gallo ${ }^{2}$, V. C. Pandolfelli ${ }^{1}$ \\ ${ }^{I}$ Universidade Federal de S. Carlos - DEMa \\ Rod. Washington Luiz, km 235, C.P. 676, 13565-905, S. Carlos, SP \\ jr@polvo.ufscar.brou vicpando@power.ufscar.br \\ ${ }^{2}$ Alcoa Alumínio S.A.
}

\begin{abstract}
Resumo
A crescente evolução tecnológica aliada ao aumento na utilização de concretos refratários têm estimulado o aprofundamento nos estudos sobre as relações granulometria/reologia nesses materiais. Normalmente, tais estudos são baseados em distribuições onde a extensão granulométrica é constante e o diâmetro máximo de partícula é próximo a $4750 \mu \mathrm{m}$. Entretanto, diferentes aplicações podem requerer a variação desse diâmetro, visando a obtenção de propriedades específicas. Sendo assim, o objetivo desse trabalho foi avaliar o impacto da extensão e distribuição granulométrica na reologia de concretos refratários zero-cimento $\left(100 \% \mathrm{Al}_{2} \mathrm{O}_{3}\right)$. Composições foram formuladas segundo o modelo de Andreasen, variando-se os coeficientes de distribuição $(q=0,21 ; 0,26$ e 0,31$)$ e os diâmetros máximos de partícula $\left(D_{L}=2800,4750\right.$ e $\left.8000 \mu \mathrm{m}\right)$. As avaliações foram realizadas em um reômetro desenvolvido para concretos refratários e os resultados revelaram uma elevada sensibilidade destes materiais frente às variações granulométricas estudadas, principalmente quando submetidos a ensaios que permitiram simular seu transporte por bombeamento.

Palavras-chave: concretos refratários, granulometria, reologia, bombeamento, diâmetro máximo.
\end{abstract}

Abstract

The growing technological demand combined with the increase application of refractory castable, has promoted further studies on the rheology of this material. As the rheological behavior is intrinsically linked to the particle size distribution, several analysis have been made aiming to determine the relationship between rheology and the particle size distribution. Usually, such analyses are based on distributions where the particle size range is constant and the maximum particle diameter is close to $4750 \mu \mathrm{m}$. However, in order to obtain specific properties, different applications may require changes of this diameter. Due to such needs, the objective of this paper was to evaluate the impact of the range and particle size distribution on the rheology of zero-cement $\left(100 \% \mathrm{Al}_{2} \mathrm{O}_{3}\right)$ refractory castable. Castables compositions were formulated according to the Andreasen model, varying the distribution coefficients $(q=0,21 ; 0,26$ and 0,31$)$ and the particles maximum diameters $\left(D_{L}=2800,4750\right.$ and $8000 \mu \mathrm{m})$. The evaluations were performed using a rheometer developed for refractory castable. The results revealed a high sensibility of these materials to variations on the particle size distributions studied, mainly when tested under conditions that simulated its pumpability.

Keywords: refractory castables, particle size distribution, rheology, pumping, maximum diameter.

\section{INTRODUÇÃO}

O contínuo avanço da indústria siderúrgica tem demandado similar evolução no desempenho dos refratários. Os concretos, em especial, vêm sendo amplamente utilizados nos últimos anos por associarem rapidez, facilidade de aplicação e desempenho similar aos materiais previamente sinterizados.

Tendo em vista que sua instalação se dá no estado fluido, o estudo do comportamento reológico dos concretos é a base para seu aperfeiçoamento tecnológico. Tradicionalmente, a reologia tem sido avaliada através do ensaio de fluidez, que por estar associado a sua tensão de escoamento [1], define o modo de moldagem do material (fluência-livre, vibração, entre outros). Contudo, este tipo de ensaio não permite avaliar o comportamento dos concretos sob cisalhamento, condição necessária para a determinação da maneira mais adequada de aplicação e transporte do mesmo (bombeamento, projeção, etc).

Uma vez que sua reologia está intrinsecamente ligada a distribuição granulométrica, vários estudos [2-4] têm sido realizados com o objetivo de entender tais relações. Esses trabalhos são fundamentados em alterações de coeficientes de distribuições acumuladas e modificações no projeto das curvas discretas, mantendo geralmente o mesmo intervalo de variação de diâmetro $(0,1$ a $4750 \mu \mathrm{m})$, ou seja, a mesma extensão granulométrica. Por outro lado, o desenvolvimento de materiais e aplicações específicas podem requerer a variação desse intervalo.

A literatura considera os concretos como materiais bifásicos [5], uma vez que são compostos por matriz (domínio dos fenômenos de superfície) e agregados (domínio dos fenômenos de massa). A variação da extensão granulométrica e do coeficiente de distribuição granulométrica segundo o modelo de Andreasen, normalmente altera o quociente matriz/agregado. 
Tal fato pode modificar a relação de domínios de forças internas que agem nesses materiais.

A redução do coeficiente de Andreasen e do diâmetro máximo de partícula geralmente aumenta o teor de matriz, resultando em concretos dominados por forças de origem superficial. Por outro lado, o aumento dessas variáveis reduz o teor de matriz com conseqüente aumento no teor de agregados, deixando o concreto mais susceptível aos fenômenos de massa. Essas variações de forças influenciam tanto as características de fluidez destes materiais quanto seu comportamento reológico em fluxo.

Deste modo, este estudo teve como objetivo avaliar o impacto da extensão e distribuição granulométrica no comportamento reológico dos concretos refratários. Para as avaliações foi utilizado um reômetro especialmente desenvolvido para esses materiais, onde foi possível acompanhar as solicitações de cisalhamento na qual foram submetidos. Os ensaios foram ainda realizados sob restrição volumétrica, condição que visou simular o processo de transporte por bombeamento.

\section{MATERIAIS E MÉTODOS}

Para a realização do estudo foram formuladas nove composições de concretos zero-cimento (100\% $\left.\mathrm{Al}_{2} \mathrm{O}_{3}\right)$ [6], baseadas no modelo de Andreasen [2-4], com variações no coeficiente de empacotamento $(q=0,21 ; 0,26$ e 0,31$)$ e no diâmetro

Tabela I - Características gerais dos concretos: porosidade teórica $\left(\mathrm{P}_{0}\right)$, área superficial volumétrica (VSA) e teor de matriz em porcentagem em volume.

[Table I - General features of the castables: theoretical porosity $\left(P_{0}\right)$, volumetric surface area (VSA) and matrix content (vol. \%).]

\begin{tabular}{lcccc}
\hline $\mathrm{q}$ & $\mathrm{D}_{\mathrm{L}}$ & $\mathrm{P}_{0}$ & $\mathrm{VSA}$ & $\%$ vol. \\
& $(\mu \mathrm{m})$ & $(\%)$ & $\left(\mathrm{m}^{2} / \mathrm{cm}^{3}\right)$ & matriz \\
\hline \multirow{2}{*}{0,21} & 2800 & 11,64 & 9,05 & 49,0 \\
\cline { 2 - 5 } & 4750 & 9,67 & 7,43 & 37,7 \\
\cline { 2 - 5 } & 8000 & 10,28 & 7,58 & 39,5 \\
\hline \multirow{2}{*}{0,26} & 2800 & 10,29 & 7,48 & 38,1 \\
\cline { 2 - 5 } & 4750 & 9,67 & 6,67 & 33,1 \\
\cline { 2 - 5 } & 8000 & 8,07 & 6,29 & 30,2 \\
\hline \multirow{3}{*}{0,31} & 2800 & 11,54 & 6,44 & 32,3 \\
\cline { 2 - 5 } & 4750 & 9,63 & 5,74 & 28,2 \\
\cline { 2 - 5 } & 8000 & 9,06 & 5,28 & 24,9 \\
\hline
\end{tabular}

Obs: VSA calculada a partir das áreas superficiais específicas das matérias-primas (B.E.T.). $\mathrm{P}_{0}$ calculado segundo o modelo de Westman e Hugill [3]. Matriz (partículas $<100 \mu \mathrm{m}$ ): aluminas calcinadas A1000 SG e A3000 FL (Alcoa - EUA); agregados (partículas $>100 \mu \mathrm{m}$ ): alumina eletrofundida branca (Alcoa-Brasil).

[Note: VSA calculated from raw-materials specific surface area (B.E.T). $P_{o}$ calculated using the Westman and Hugill model [3]. Matrix (particles $<100 \mu \mathrm{m}$ ): calcined aluminas A1000 SG and A3000 FL (AlcoaUSA); aggregates (particles $>100 \mu \mathrm{m}$ ): white fused alumina (AlcoaBrazil).
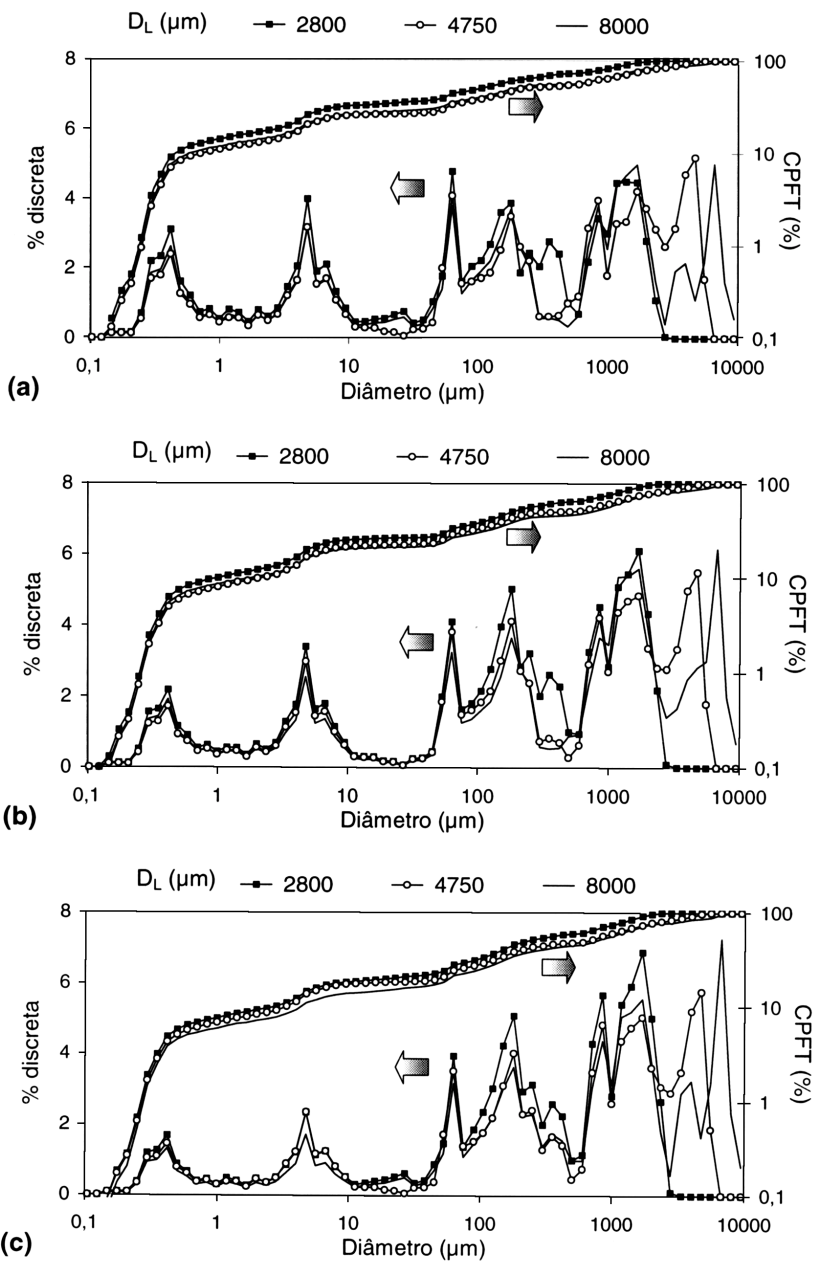

Figura 1: Curvas de distribuições granulométricas discretas e acumuladas das composições formuladas com diferentes diâmetros máximos de partícula $\left(D_{L}=2800,4750\right.$ e $\left.8000 \mu \mathrm{m}\right)$ e coeficientes de Andreasen (q): (a) 0,21 ; (b) 0,26 e (c) 0,31 .

[Figure 1: Cumulative and discrete particle size distributions for the compositions formulated with different maximum particle size diameters $\left(D_{L}=2800,4750\right.$ and $\left.8000 \mu \mathrm{m}\right)$ and Andreasen coefficients (q): (a) 0.21 , (b) 0.26 and (c) 0.31.$]$

máximo de partícula $\left(\mathrm{D}_{\mathrm{L}}=2800,4750\right.$ e $\left.8000 \mu \mathrm{m}\right)$. As características das composições se encontram na Tabela I e suas distribuições granulométricas nas Figs. 1(a), 1(b) e 1(c).

Fixou-se o teor de água em $15 \%$ em volume e a dispersão foi feita com ácido cítrico no teor de $0,26 \mathrm{mg} / \mathrm{m}^{2}$. A mistura foi realizada em um reômetro [7] a uma rotação constante de $33 \mathrm{rpm}$ e a adição de água ocorreu em duas etapas, segundo procedimentos desenvolvidos em [8]. Logo após o término da mistura, foi medida a fluidez das composições segundo a norma ASTM C-860 adaptada para concretos auto-escoantes.

$\mathrm{Na}$ Tabela II são apresentadas algumas propriedades dos concretos após a adição total da água. O cálculo da concentração volumétrica de sólidos da matriz foi feito considerando-se a suspensão formada por água + partículas menores que $100 \mu \mathrm{m}$. O valor de IPS corresponde a distância média de separação entre as partículas da matriz e o MPT a distância média entre os agregados.

Em seguida, fez-se a determinação do comportamento 
Tabela II - Concentração volumétrica de sólidos na matriz $\left(\phi_{\text {matriz }}\right)$ além dos respectivos valores de distância média de separação entre partículas da matriz [2, 3] (IPS) e dos agregados [3, 9] (MPT).

[Table II - Matrix volumetric concentration of solids $\left(\phi_{\text {matrix }}\right)$ and mean separation distance between matrix particles [2, 3] (IPS) and coarse aggregates [3, 9] (MPT).]

\begin{tabular}{lllll}
\hline $\mathrm{q}$ & $\mathrm{D}_{\mathrm{L}}$ & $\phi_{\text {matriz }}$ & IPS & MPT \\
& $(\mu \mathrm{m})$ & $(\%)$ & $(\mu \mathrm{m})$ & $(\mu \mathrm{m})$ \\
\hline \multirow{2}{*}{0,21} & 2800 & 73,01 & 0,018 & 113,66 \\
\cline { 2 - 5 } & 4750 & 67,49 & 0,032 & 105,62 \\
\cline { 2 - 5 } & 8000 & 68,56 & 0,028 & 108,72 \\
\hline \multirow{2}{*}{0,26} & 2800 & 67,62 & 0,031 & 71,50 \\
\cline { 2 - 5 } & 4750 & 64,43 & 0,042 & 82,51 \\
\cline { 2 - 5 } & 8000 & 62,38 & 0,048 & 82,91 \\
\hline \multirow{2}{*}{0,31} & 2800 & 63,84 & 0,042 & 56,51 \\
\cline { 2 - 5 } & 4750 & 60,62 & 0,052 & 67,85 \\
\cline { 2 - 5 } & 8000 & 57,68 & 0,065 & 63,69 \\
\hline
\end{tabular}

Obs: $\phi_{\text {matriz }}=$ Concentração volumétrica de sólidos na suspensão que compõe a matriz: água + partículas $<100 \mu \mathrm{m}$.

[Note: $\phi_{\text {matriz }}=$ Volumetric solid content in the matrix suspension: water + particles $<100 \mu \mathrm{m}$.]

reológico dos concretos por meio da realização de dois ciclos de cisalhamento sucessivos (entre $2 \mathrm{rpm}$ e $75 \mathrm{rpm}$ ), sendo que no segundo foi utilizado um dispositivo para restrição volumétrica. Foi possível obter através dos ciclos o valor de torque das composições em altas rotações (75 rpm).

\section{RESULTADOS E DISCUSSÃO}

As características de moldagem de um concreto são normalmente influenciadas pela fluidez do mesmo [2, 3]. Composições formuladas com $\mathrm{q}=0,21$ atingiram elevados níveis de fluidez se comportando como concretos auto-escoantes, independente do diâmetro máximo de partícula (Fig. 2). A imprecisão das medidas obtidas no ensaio de mesa de fluidez $[1,7]$ permite inferir que, apesar da composição com diâmetro máximo $4750 \mu \mathrm{m}$ estar pouco abaixo da região de autoescoamento, esta pode ser considerada um concreto autoescoante.

Os níveis de fluidez atingidos pelos concretos com $\mathrm{q}=0,21$ se devem aos seus elevados teores de matriz [2, 3] (Tabela I) e MPT (Tabela II). Tais propriedades distanciam os grãos grossos no movimento da fluidez e reduzem a interferência entre os mesmos, deixando o sistema sujeito a forças de origem superficial advindas da matriz [2-4].

Os concretos formulados com $q=0,26$ comportaram-se como vibráveis (Fig. 2) no teor de água utilizado, apresentando valores de fluidez intermediários entre as composições com q = 0,21 e 0,31 . Concretos com esse coeficiente $(q=0,26)$ são denominados na literatura como multifuncionais [10], pois o controle da fluidez pode ser feito através do teor de água utilizado em seu processamento.

Ao contrário das composições com $\mathrm{q}=0,21$; o diâmetro

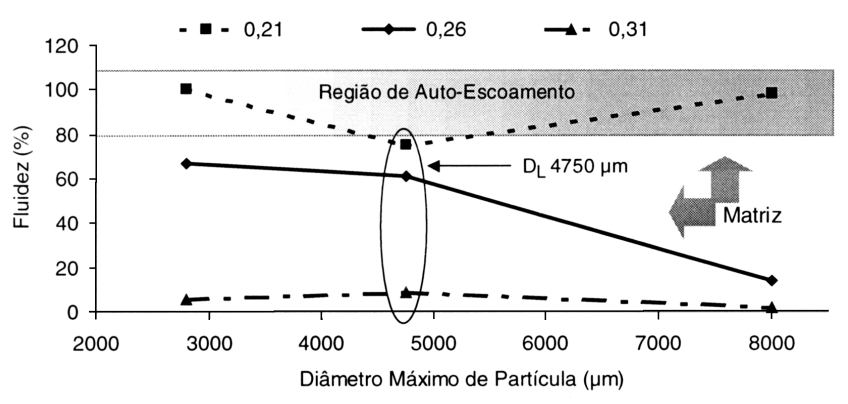

Figura 2: Fluidez medida após a mistura em função do diâmetro máximo de partícula para as composições formuladas com diferentes coefícientes de Andreasen $(q=0,21 ; 0,26$ e 0,31$)$. Obs: as setas indicam o sentido de aumento no teor de matriz dos concretos.

[Figure 2: Free flow values measured after mixing as a function of the largest particle diameter for the castables formulated with different Andreasen coefficients $(q=0.21 ; 0.26$ and 0.31$)$. Arrows indicate an increase in castable matrix content.]

máximo de partícula influenciou significativamente a fluidez nos concretos com coeficiente de Andreasen $q=0,26$, onde o aumento dessa variável resultou em composições menos fluidas (Fig. 2). Isso ocorre possivelmente devido a redução no teor de matriz com o aumento de $\mathrm{D}_{\mathrm{L}}$ (Tabela I), causando aumento da contribuição dos fenômenos de massa originados dos grãos grossos e redução da capacidade de lubrificação entre os mesmos.

Nas composições com $q=0,31$, o reduzido teor de matriz resultou em concretos com valores de fluidez próximos a zero, independentemente do valor de $\mathrm{D}_{\mathrm{L}}$.

Resultados anteriores [11] mostram que concretos formulados com $\mathrm{D}_{\mathrm{L}}=4750 \mu \mathrm{m}, \mathrm{q}=0,21$ e processados com $15 \%$ em volume de água, apresentaram níveis de fluidez propícios a segregação (maiores que 120\%); os quais neste trabalho se mostraram auto-escoantes ( $\sim 78 \%$ de fluidez). Tal fato atesta a importância das curvas discretas de distribuição e a imprecisão das curvas acumuladas, uma vez que para um mesmo coeficiente de Andreasen, os níveis de fluidez variaram significativamente em comparação com valores da literatura.

Após a análise da fluidez, propriedade que indica o modo de moldagem dos concretos, efetuou-se uma caracterização sob cisalhamento. Estes ensaios, além de avaliarem o comportamento reológico das composições, são capazes de fornecer valores de torque em baixas e altas rotações, informações importantes para a determinação do processo de transporte no qual deve ser submetido um concreto.

Nos gráficos das Figs. 3, 4 e 5 são apresentados os ciclos de cisalhamento dos concretos com diferentes coeficientes de Andreasen e diâmetros máximos de partícula, tanto em livre mobilidade das partículas, como sob restrição volumétrica.

Analisando inicialmente os concretos com q $=0,21$, observase no gráfico da Fig. 3 que no ciclo realizado sem restrição volumétrica, o concreto com maior nível de esforço em altas rotações é o de menor $\mathrm{D}_{\mathrm{L}}(2800 \mu \mathrm{m})$. A elevada concentração 
de sólidos na matriz ( $\phi_{\text {matriz }}=73,01 \%$ ) faz com que suas partículas estejam muito próximas entre si (IPS $=0,018 \mu \mathrm{m}$ ) dificultando sua mobilidade em altas rotações. Essa dificuldade de movimentação foi intensificada no ensaio realizado sob restrição volumétrica (Fig. 3), uma vez que em altas rotações ocorreu transição de comportamento newtoniano (sem restrição) para dilatante (volume restrito). $\mathrm{O}$ alto valor de torque apresentado por esse concreto em altas rotações é mostrado em detalhes no gráfico da Fig. 6.

Com relação aos concretos restantes desse sistema $(\mathrm{q}=$ 0,21), é possível observar nas Tabelas I e II similaridades de propriedades entre essas duas composições $\left(D_{L}=4750\right.$ e 8000 $\mu \mathrm{m})$. Tal fato possivelmente ocasionou a semelhança entre suas curvas de cisalhamento (comportamento pseudoplástico), principalmente quando o ensaio foi realizado sob restrição volumétrica (Fig. 3).

Adicionalmente, foram observados reduzidos valores de

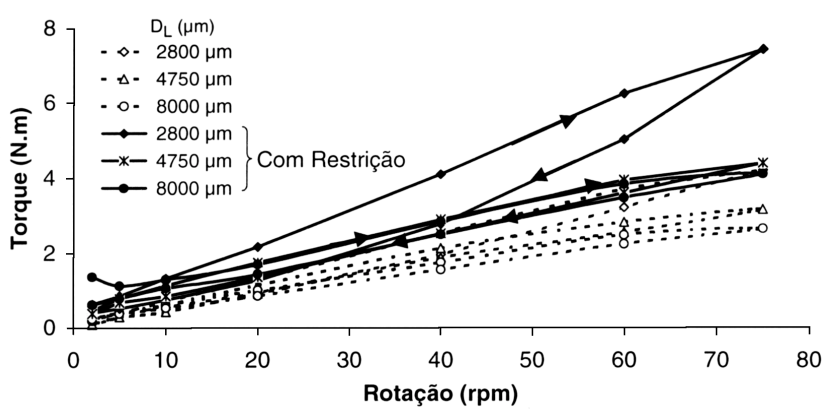

Figura 3: Ciclos de cisalhamento realizados nos concretos com coeficiente de empacotamento de Andreasen $\mathrm{q}=0,21$ e diferentes diâmetros máximos de partícula $\left(\mathrm{D}_{\mathrm{L}}=2800 ; 4750\right.$ e $\left.8000 \mu \mathrm{m}\right)$. Obs: Condição de livre mobilidade das partículas é representada por linhas tracejadas enquanto sob restrição volumétrica por linhas cheias.

[Figure 3: Shearing cycles for castables with Andreasen coefficient $q=$ 0.21 and different maximum aggregate diameters $\left(D_{L}=2800 ; 4750\right.$ and $8000 \mu \mathrm{m})$. Note: Unrestricted shearing - doted lines, restricted cycle-full lines.]

torque em baixas rotações no sistema com $q=0,21$ (Fig. 3). Esses valores são condizentes com a literatura e tem comportamento inverso ao da fluidez [11] (Fig. 2).

Os resultados obtidos para os concretos com $q=0,26$ mostram que o menor teor de matriz desse sistema, em relação ao $\mathrm{q}=0,21$, permite o equilíbrio entre forças mássicas, devidas à presença dos agregados, e superficiais, advindas da matriz [2-4]. O aumento de $\mathrm{D}_{\mathrm{L}}$ em concretos formulados com esse coeficiente elevou os níveis de torque em altas rotações, tanto para o ciclo realizado sem restrição, quanto para o de volume restrito (Figs. 4 e 6). Com o aumento de $\mathrm{D}_{\mathrm{L}}$ (redução no teor de matriz) verifica-se uma diminuição nas forças de origem superficial e conseqüente aumento nos fenômenos mássicos. Desta maneira a interferência entre os agregados é intensificada, elevando os níveis de torque.

Quanto aos valores de torque em baixas rotações, os concretos com $q=0,26$ apresentaram níveis intermediários entre as composições com $q=0,21$ (Fig. 2) e q =0,31 (Fig. 4), fato que justifica seu comportamento vibrável de moldagem (Fig. 1), para

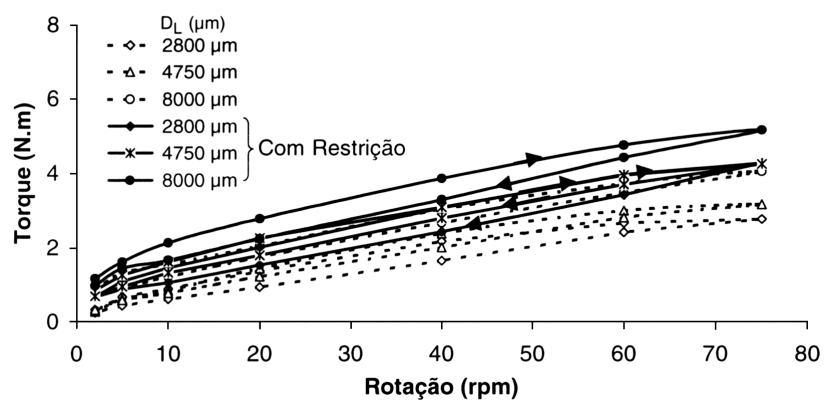

Figura 4: Correlação entre torque e rotação para as composições com coeficiente de empacotamento de Andreasen $\mathrm{q}=0,26$ e diferentes diâmetros máximos de partícula $\left(\mathrm{D}_{\mathrm{L}}=2800 ; 4750\right.$ e $\left.8000 \mu \mathrm{m}\right)$.

[Figure 4: Relationship between torque and rotation for compositions with Andreasen coefficient $q=0.26$ and different maximum particle size diameters $\left(D_{L}=2800 ; 4750\right.$ and $\left.8000 \mu \mathrm{m}\right)$.]

a quantidade de água empregada nesse trabalho.

Concretos formulados com coeficiente de Andreasen $\mathrm{q}=$ 0,31 geralmente são dominados em quase toda a sua extensão por fenômenos de massa, em virtude do reduzido teor de matriz. Tal fato justifica o maior nível de esforço em altas rotações observado no concreto com $\mathrm{D}_{\mathrm{L}}$ igual a $4750 \mu \mathrm{m}$ em relação ao concreto com $2800 \mu \mathrm{m}$, quando o ciclo é realizado sem restrição de volume (Figs. 5 e 6); uma vez que o aumento de $\mathrm{D}_{\mathrm{L}}$ também reduz o teor de matriz.

O reduzido valor de torque apresentado pela composição q $=0,31$ com $\mathrm{D}_{\mathrm{L}}=8000 \mu \mathrm{m}$ é justificado pela perda de coesão desse concreto. Fato ocorrido possivelmente em virtude do seu baixo teor de matriz (Tabela I).

Quando o ensaio é realizado sob volume restrito é verificada elevada sensibilidade da composição com $\mathrm{D}_{\mathrm{L}}=2800 \mu \mathrm{m}$. O valor de IPS nesse concreto $(0,042 \mu \mathrm{m})$ juntamente com sua concentração volumétrica de sólidos $\left(\phi_{\text {matriz }}=63,84 \%\right)$ provocaram um aumento da viscosidade da matriz em altas rotações, impossibilitando eficiência na lubrificação dos agregados. Tais fatores, somados com a restrição de volume e a reduzida distância entre grãos grossos $(\mathrm{MPT}=56,51 \mu \mathrm{m})$, intensificaram a interferência entre os mesmos, elevando os níveis de esforços (Figs. 5 e 6).

Os baixos níveis de fluidez das composições com $q=0,31$

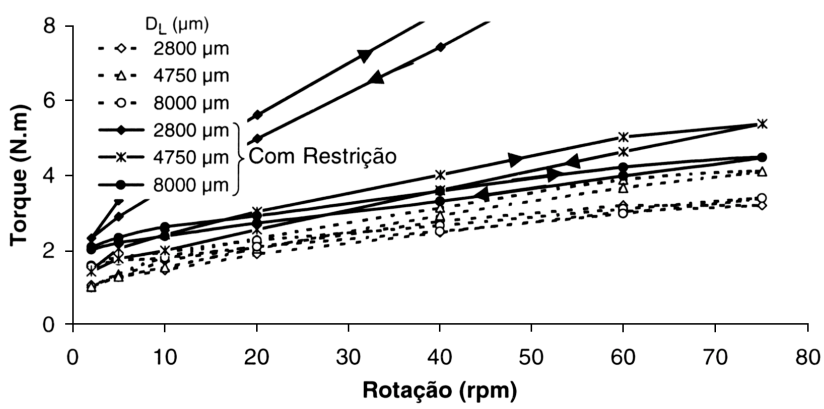

Figura 5: Torque em função da rotação para as composições com coeficiente de empacotamento de Andreasen $\mathrm{q}=0,31$ e diferentes diâmetros máximos de partícula $\left(\mathrm{D}_{\mathrm{L}}=2800,4750\right.$ e $\left.8000 \mu \mathrm{m}\right)$.

[Figure 5: Rheological behavior of the compositions with Andreasen coefficient $q=0.31$ and different maximum particle size diameters $\left(D_{L}\right.$ $=2800,4750$ and $8000 \mu \mathrm{m})$. 


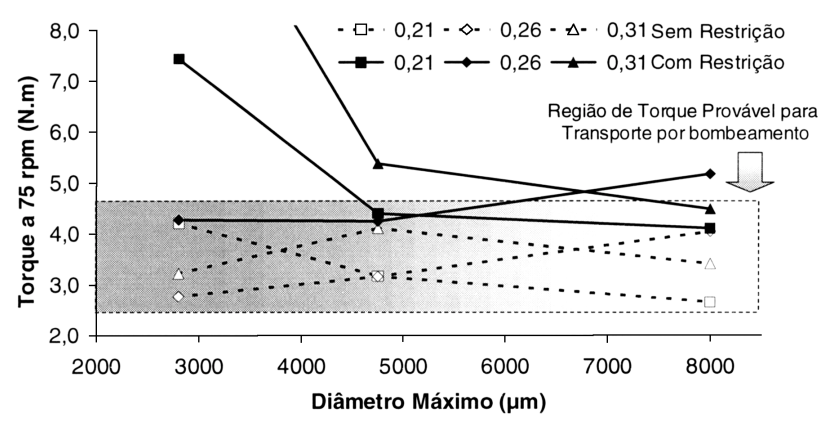

Figura 6: Correlação entre torque em altas taxas de cisalhamento (75 rpm) e diâmetro máximo de partícula para as composições estudadas, tanto para a condição de restrição volumétrica quanto para livre mobilidade das partículas.

[Figure 6: Relationship between torque at high shear rates (75 rpm) and the maximum particle size diameter for the compositions subjected to unrestricted and restricted shearing cycles.]

(Fig. 2) podem ser explicados por seus elevados valores de torque em baixas rotações [11] (Fig. 5).

Adicionalmente, pode-se identificar quais concretos analisados nesse trabalho poderiam ser submetidos ao bombeamento. Observando o gráfico da Fig. 6, nota-se uma região de torque em altas rotações ideal para o bombeamento; estabelecida segundo estudos anteriores [12]. É possível observar quatro composições que se localizam dentro dessa região quando o ensaio é realizado com restrição de volume. São as seguintes: $q=0,21$ com diâmetros máximos 4750 e 8000 $\mu \mathrm{m} ; \mathrm{q}=0,26 \mathrm{com}_{\mathrm{L}}=2800$ e $4750 \mu \mathrm{m} \mathrm{e} \mathrm{q}=0,31 \mathrm{com} \mathrm{D}_{\mathrm{L}}=8000$ $\mu \mathrm{m}$. Este último não pode ser considerado para análises posteriores, uma vez que o concreto perdeu coesão em altas taxas de cisalhamento.

Observando o comportamento reológico total destas composições, verifica-se, em geral, que todas apresentaram comportamento pseudoplástico, relativamente baixos valores de torque em baixas e altas rotações e baixa sensibilidade a restrição de volume com manutenção da coesão. Sendo assim, os concretos acima citados, exceto o $\mathrm{q}=0,31 \mathrm{com} \mathrm{D}_{\mathrm{L}}=8000 \mu \mathrm{m}$, podem possivelmente serem submetidos ao processo de transporte por bombeamento. Cabe ressaltar que estudos anteriores [11] já haviam identificado a bombeabilidade do concreto com $q=0,26$ e $D_{L}=$ $4750 \mu \mathrm{m}$, a qual foi confirmada neste trabalho.

\section{CONCLUSÕES}

$\mathrm{O}$ ensaio de fluidez se mostrou insuficiente para avaliar o comportamento reológico dos concretos, sendo este indicado somente para analisar o modo de moldagem desses materiais. Para uma avaliação reológica completa foi necessário a realização de ciclos de cisalhamento. Esses ensaios indicaram o comportamento reológico dos concretos sob fluxo, além de terem fornecido informações dos valores de torque em baixas e altas taxas de cisalhamento. Tais ensaios permitiram concluir que a extensão e distribuição granulométrica são variáveis que influenciam significativamente o comportamento reológico dos concretos.

Foi observada a importância das curvas discretas de distribuição granulométrica e a imprecisão das curvas acumuladas no controle da fluidez de concretos refratários.
A redução do coeficiente de Andreasen e do diâmetro máximo de partícula resultou em concretos com maior teor de matriz, sendo influenciados em quase toda a sua extensão por fenômenos de superfície. Por outro lado, o aumento dessas variáveis gerou concretos com maior influência dos fenômenos de massa.

Os resultados obtidos possibilitaram a identificação de novas composições de concretos bombeáveis com diâmetros máximos de partículas diferentes dos encontrados em literatura. Deste modo, comportamentos reológicos distintos podem se adequar a diferentes técnicas de aplicação, moldagem e desempenho em uso.

\section{AGRADECIMENTOS}

Os autores agradecem à FAPESP, ao CNPq è̀ Alcoa Alumínio S.A. pelo apoio recebido durante a realização deste trabalho.

\section{REFERÊNCIAS}

[1] K. Watanabe, M. Ishikawa, M. Wakamatsu, "Rheology of castables refractories", Taikabutsu Overseas 9, 1 (1989) 41.

[2] R. G. Pileggi, V. C. Pandolfelli, "Reflexões sobre distribuição granulométrica e sua correlação com a reologia de concretos refratários", Ceramic News Special South America (1999) 6.

[3] I. R. Oliveira, A. R. Studart, R. G. Pileggi, V. C. Pandolfelli, Dispersão e empacotamento de partículas - princípios básicos e aplicações no processamento cerâmico. Fazendo Arte Editorial (2000) $224 \mathrm{p}$.

[4] R. G. Pileggi, "Ferramentas para o estudo e desenvolvimento de concretos refratários", Tese de Doutorado, UFSCar, S. Carlos, SP(2001).

[5] I. B. Topcu, F. Kocatasin, "A two-phase composite material approach to the workability of concrete", Cement and Concrete Composites 17 (1995) 319.

[6] A. R. Studart, R. G. Pileggi, W. Zhong, V. C. Pandolfelli, "Processing of zero-cement self-flow alumina castable", Am. Ceram. Soc. Bull. 77, 12 (1998) 60.

[7] R. G. Pileggi, V. C. Pandolfelli, A. E. Paiva, J. Gallo, "Novel rheometer for refractory castables", Am. Ceram. Soc. Bull. 79, 1 (2000) 54 .

[8] R. G. Pileggi, A. R. Studart, V. C. Pandolfelli, "How mixing affects the rheology of refractory castables", Am. Ceram. Soc. Bull. 80, 6 (2001) 27; 80, 7 (2001) 38.

[9] P. Bonadia, R. G. Pileggi, A. R. Studart, S. L. Vendrasco, V. C. Pandolfelli, "Applying MPT principle to high-alumina castable", Am. Ceram. Soc. Bull. 78, 3 (1999) 57.

[10] A. R. Studart, R. G. Pileggi, J. Gallo, V. C. Pandolfelli, "High alumina multifuncional refractory castables", Am. Ceram. Soc. Bull. 80, 11 (2001) 34.

[11] R. G. Pileggi, V. C. Pandolfelli, "Rheology and particle size distribuition of pumpable refractory castables", Am. Ceram. Soc. Bull. 80, 10 (2001) 52.

[12] R. G. Pileggi, A. R. Studart, C. Pagliosa Neto, V.C. Pandolfelli, "Caracterização reológica de concretos refratários bombeáveis", Cerâmica 46, 300 (2000) 176.

(Rec. 28/03/02, Rev. 02/07/02, Ac. 21/07/02) 